\title{
Hipotiroidismo Subclínico como factor de riesgo para enfermedad vascular
}

Subclinical Hypothyroidism Is an Independent Risk Factor for Atherosclerosis and Myocardyal Infarction in Elderly Women: The Rótterdam Study. Hak AE; Pols HAP; Visser TJ; Drexhage HA; Hofman A; Witteman JCM. Ann Intern Med. 2000; 132:270-277.

\section{Objetivo}

Investigar si el hipotiroidismo subclínico (HTS) y la autoinmunidad tiroidea están asociados a aterosclerosis (AS) aórtica e infarto agudo de miocardio (IAM) en mujeres posmenopáusicas.

\section{Diseño}

Estudio poblacional de corte transversal.

\section{Lugar}

Un distrito de Rotterdam, Holanda

\section{Pacientes}

Muestra aleatoria de 1149 mujeres con una media de edad de 69 años, participantes de una cohorte sobre prevalencia de enfermedades crónicas (estudio Rotterdam).

\section{Evaluación de factores de riesgo}

Se definió HTS mediante tirotrofina (TSH) Lumitest con valores mayores a $4 \mathrm{mU} / \mathrm{L}$ con tiroxina libre normal; la presencia de AS aórtica mediante evaluación de Radiografía de perfil lumbar con evidencia de depósitos de calcio en aorta abdominal; y la presencia de Infarto de miocardio al inicio del estudio (1990-93) y durante seguimiento hasta el 1 de Abril de 1996, mediante autoreporte, evaluación electrocardiográfica y revisión de historias clínicas.

\section{Medición de resultados principales}

Se obtuvo el valor de TSH en 1149 pacientes. Luego de la aplicación de los criterios de exclusión (enfermedad tiroidea, uso de medicación que afecte la función tiroidea, datos o seguimiento inadecuados) se incluyeron 975 pacientes para evaluación de AS aórtica, 994 para evaluación de IAM al inicio, y 1036 para incidencia de IAM durante el seguimiento.

Se realizaron análisis multivariables* ${ }^{*}$ para evaluar la asociación entre hipotiroidismo subclínico y la presencia de AS aórtica o IAM ajustando por la presencia de obesidad, tabaquismo, presión arterial, colesterol y uso de $\beta$ bloqueantes .

\section{Resultados Principales}

La prevalencia de HTS fue de $10.8 \%$. No hubo diferencias en cuanto a edad, índice de masa corporal (IMC), TA y hábito tabáquico entre las mujeres eutiroideas y las mujeres con HTS.

EI HTS se asoció con mayor riesgo de AS aórtica OR* 1.7 (IC 95\% 1.1 a 2.6) y de IAM OR 2.3 (IC 95\% 1.3 a 4.0). Durante el seguimiento de 4.6 años se detectaron 16 nuevos IAM; el RR para IAM en las mujeres con HTS fue de 2.5 (IC 0.7 a 9.1, p=NS). La presencia de anticuerpos antitiroideos incrementó el riesgo de AS aórtica OR 1.9 (IC 95\% 1.1 a 3.6) y de IAM, OR 3.1 (IC 95\% 1.5 a 16.3) en las mujeres con HTS, pero no en las mujeres eutiroideas.

\section{Conclusiones}

EI HTS es una entidad prevalente entre mujeres mayores, y se asocia con mayor frecuencia de AS aórtica y IAM.

\section{Comentario}

El estudio Rotterdam fue diseñado para evaluar la ocurrencia y los factores determinantes de enfermedades crónicas en la población mayor. Este trabajo se realizó sobre una muestra aleatoria de mujeres participantes de este estudio. El hecho de tratarse de un estudio de corte transversal podría ser la primera limitación, como aclaran incluso los mismos autores. Los resultados obtenidos parecen ser representativos, con prevalencias similares a las reportadas en otros trabajos $1,2,3$.

Además de relacionar al HTS con el IAM, este es el primer estudio que describe también una asociación entre el HTS y la AS, sugiriendo un mecanismo aterogénico en la génesis del IAM en estos pacientes. Otro hallazgo es la mayor asociación entre el HTS y AS o IAM ante la presencia de anticuerpos antitiroideos, lo que suma nueva evidencia a favor de la relación entre la autoinmunidad tiroidea y la enfermedad coronaria. Con respecto al componente prospectivo del estudio, a pesar de ser similares a los obtenidos en el componente transversal, los resultados obtenidos no fueron significativos probablemente por la baja tasa de eventos registrados.

En el presente trabajo la población esta compuesta por mujeres con una media de edad de 68.9 años, y la prevalencia de HTS encontrada fue de $10.8 \%$.

Es interesante resaltar que no se encontraron diferencias significativas en el Indice de Masa Corporal de las mujeres con HTS y las eutiroideas, a pesar de que frecuentemente se le atribuye al HTS el exceso de peso de muchas pacientes.

Si tenemos en cuenta la prevalencia de HTS en este tipo de pacientes, el riesgo atribuible al HTS sería similar al de otros factores de riesgo cardiovascular considerados "clásicos". De todos modos será necesario confirmar esta asociación en estudios de seguimiento antes de incorporar al HTS como marcador de riesgo cardiovascular.

\section{Dr. Hugo Villafañe [ Médico General. Hospital Italiano de Córdoba ]}

